\title{
ARTIGOS
}

\section{PROPUESTA DE INDICADORES DE GESTIÓN PARA LA EDUCACIÓN PÚBLICA LOCAL CHILENA}

SEBASTIAN DONOSO-DÍAZI

GENARA DÍAZ LÓPEZ"

NIBALDO BENAVIDES-MORENO"I

\section{RESUMEN}

El texto precisa indicadores claves para la gestión de los sistemas educativos locales de la educación pública chilena. Materia sustantiva dado que ha sido constantemente señalada como una carencia importante y recurrente de este sector. Por ende, se necesitan avances en este ámbito, más aún dado que el año 2018 comenzó el traspaso gradual de la institucionalidad de la educación local hacia una nueva modalidad de organización (los Servicios Locales de Educación), causal relevante para corregir los problemas detectados, en el entendido que es posible a partir de su correcta medición y procesamiento. Sobre la base de una metodología cualitativa, basada en la consulta a informantes claves de las unidades de gestión de la educación pública local, se identifican y validan algunos indicadores, proveyéndose un conjunto de variables de simple aplicación, que permite a estas unidades mejorar su información para la mejor gestión.

GESTIÓN・ADMINISTRACIÓN LOCAL・EDUCACIÓN PÚBLICA・INDICADORES

\section{MANAGEMENT INDICATORS PROPOSAL FOR LOCAL PUBLIC EDUCATION IN CHILE}

\section{ABSTRACT}

The study specifies key indicators for managing local education systems of public education in Chile, as there has been significant and recurring shortages in this sector. Therefore, it is necessary to move forward in this area, especially because the gradual transfer of the institutionality of the local education authority to

Universidad de Talca, Instituto de Investigación y Desarrollo Educacional, Chile; sdonoso@uta/ca.cl del Maule, Chile: donoso.seb@gmail.com

\section{III}

Universidad de Talca, Instituto de Investigación y Desarrollo Educacional, Chile;nbenavides@utalca.cl a new form of organization (Local Education Services) started in 2018.This transfer is extremely relevant to solve the problems detected, asthat can be done through correctly measuring and processing the problems. Based on a qualitative methodology, which in its turn was based on consultation with the main units for local public management education, some indicators are identified and validated, providing a set of variables of simple application, which allow these units to improve their information for better management. 


\section{PROPOSITION D'INDICATEURS DE GESTION POUR L'ÉDUCATION PUBLIQUE LOCALE CHILIENNE}

RÉSUMÉ

Ce travail examine les indicateurs clé de la gestion infranationale des systèmes éducatifs de l'éducation publique chilienne. Cette question de fond est constamment mise en avant comme une carence importante et récurrente du secteur. Il est donc nécessaire d'avancer dans ce domaine, une fois que 2018 marque le début du transfert progressif de l'institutionnalisation de l'administration scolaire locale vers une nouvelle modalité d'organisation (les Services d'Éducation Locale). Cette modalité s'avère très pertinent pour corriger les problèmes détectés, dans la mesure où sa dimension et son traitement sont analysés correctement. Cette recherche repose sur une méthodologie qualitative basée sur une consultation des principales unités de gestion de l'éducation publique locale. Certains indicateurs ont été identifiés et validés offrant un ensemble de variables d'application simple à fin de permettre à ces unités d'améliorer leurs informations en vue d'une gestion meilleure.

GESTION • ADMINISTRATION LOCALE • ÉDUCATION PUBLIQUE • INDICATEURS

\section{PROPOSTA DE INDICADORES DE GESTÃO PARA A EDUCAÇÃO PÚBLICA LOCAL CHILENA}

RESUMO

O texto especifica indicadores-chave para a gestão dos sistemas educativos locais da educação pública chilena. Matéria substantiva, pois tem sido constantemente apontada como uma carência importante e recorrente desse setor. Portanto, é preciso avançar nessa área, especialmente porque em 2018 foi iniciada a transferência gradual da institucionalidade da educação local para uma nova forma de organização (os Serviços de Educação Local), sumamente relevante para corrigir os problemas detectados, no entendimento de que isso é possivel a partir de sua medição e processamento corretos. Com base em uma metodologia qualitativa, baseada na consulta às principais unidades de gestão da educação pública local, alguns indicadores são identificados e validados, fornecendo um conjunto de variáveis de simples aplicação, que permite que essas unidades melhorem suas informações para a melhor gestão. 


\section{LA GESTIÓN DE LA EDUCACIÓN PÚBLICA SUBNACIONAL Y LAS DEMANDAS DE MODERNIZACIÓN DEL SECTOR}

A EdUCACIÓN PÚBlica A PARTIR DE LA REFORMA DE1980, SUSTENTADA EN UNA visión de mercado neoliberal, manifiesta dos problemáticas sustantivas. La primera, un déficit importante en la eficiencia de la gestión de los Departamento de Administración de Educación Municipal (en adelante DAEM$^{1}$ ), como entes responsables de su implementación, esencialmente respecto del manejo de los recursos, visión cortoplacista sobre el panorama educativo comunal y de sus establecimientos escolares (MARCEL; RACZYNSKI, 2009; GONZÁLEZ; GONZÁLEZ; GALDAMES, 2015), situación que ha acentuado las dificultades de gestión de la educación pública local en Chile, provocando adicionalmente que ésta no cumpla debidamente con las expectativas y requerimientos de la sociedad (ORGANIZACIÓN PARA LA COOPERACIÓN Y EL DESARROLLO ECONÓMICO -OCDE, 2004). Aspecto que se asocia a la segunda problemática, la sistemática caída de sus indicadores educacionales convencionales de eficiencia interna y externa, a saber: la matrícula total escolar pública pasó de atender al 78\%-en1981-al 36\% de los estudiantes el año 2017 (OCDE, 2017a), e igualmente experimentó un complejo tránsito de los resultados de aprendizaje en las pruebas escolares nacionales, con impactos sustantivos en la gestión y financiamiento del 
sector, que han conformado un escenario crítico con serias implicancias sobre su viabilidad futura de mediano plazo (CHILE, 2006; BELLEI, 2015).

La reforma señalada generó transformaciones estructurales del sistema educativo, destacándose el traspaso de los establecimientos escolares (infraestructura), personal docente y administrativo desde el aparato central del Estado (Ministerio de Educación) a los municipios, proceso que se caracterizó -entre otros elementos- por ser una medida intempestiva e improvisada (WAISSBLUTH, 2013; LARRAÑAGA; PEIRANO; FALCK, 2009). En esos años el país carecía de experiencias relevante de gestión local de la educación, adoptándose por el Gobierno este camino, con medidas que no habían sido aplicadas en otras realidades, ni en esa escala de operación, traspasando el primer año al $70 \%$ de los establecimientos escolares y su totalidad un par de años después (DONOSO, 2016). Además, no consideró un acompañamiento y el apoyo del Gobierno nacional a los Municipios en la gestión, ni para adecuarse a la nueva modalidad de financiamiento vía subsidio a la demanda, ni para abordar los temas de gestión del personal, ello en un marco político diseñado para favorecer la privatización (JOFRÉ, 1988; JOIKO, 2012; BALL; YOUDELL, 2007).

La reforma en comento asignó al Municipio y específicamente al Alcalde como responsable de la gestión local de la educación pública, sin dotarla de los recursos financieros y técnicos indispensables para generar una institucionalidad consistente con la tarea de calidad por la que se le evalúa. Es decir, se han estipulado un conjunto de responsabilidades sin gestionar en estas unidades subnacionales las capacidades y recursos para enfrentarlas debidamente (ESPÍNOLA; SILVA, 2009; POLITEIA, 2008). Una muestra de la falta de rigurosidad en este proceso es que una década y media después de iniciada la reforma (en el año 1995), el Gobierno estableció el Plan Anual de Educación Municipal (en adelante PADEM) como el instrumento de planificación estratégica de la educación pública local, mecanismo de carácter obligatorio que desde entonces los Municipios deben formular en forma anual.

En la actualidad existen pocas dudas respecto de la crisis sistémica de la educación chilena, y sobre el hecho de que los movimientos estudiantiles de 2006 y 2011 tuvieron un papel decisivo al situar en la agenda pública las demandas de fortalecimiento de la educación pública escolar y superior, (DONOSO, 2013) y proponiendo orientaciones políticas que derivaron más tarde en una nueva ley que creó el Sistema de Educación Pública, cuya implementación -en forma gradual- se inicia el año 2018.

En este marco, las propuestas de fortalecimiento de la Educación Pública, sustentadas en la ley recientemente aprobada dio origen al Sistema Nacional de Educación Pública (CHILE, 2017), de progresiva aplicación a partir de este año (2018), en razón de lo cual es más 
relevante identificar indicadores claves de los procesos de control de gestión de las unidades locales de educación pública, debido a que el nuevo sistema implicará una transición de la educación local municipal hacia los Servicios Locales de Educación (SLE), fenómeno programado para desarrollarse al menos por una década. Lapso en el cual operaran ambas modalidades organizativas en forma simultánea, los municipios con sus DAEM y por otra parte los nuevos SLE que se van generando.

Adicionalmente, los nuevos SLE necesitarán generar un plan estratégico con un horizonte de seis años, por ende indistintamente de cuál sea el instrumento de planificación que emplee el DAEM o el SLE, sus elementos medulares se referirán a la implementación, con la finalidad de superar las limitaciones que posee el instrumento vigente (PADEM), para no replicarles en una escala de mayor magnitud como es el SLE. ${ }^{2}$

El PADEM es un instrumento diseñado desde la gestión pública convencional, con escaso diálogo con la gestión moderna del sector educativo, evidenciando problemas de diseño y operación con el sector en su calendario de operación, así como de sinergia con otros instrumentos de planificación comunal y con los planes de desarrollo de cada establecimiento escolar: Proyecto Educativo Institucional y Proyecto de Mejoramiento Educativo. En este aspecto la educación pública chilena está entrabada por su obsolescencia en este plano y por la desconexión de los instrumentos en uso con el contexto social, económico y político del territorio en que se sitúa (ELACQUA; MARTÍNEZ; ANINAT, 2010).

A partir del año 2000, la educación avanzó lentamente en logros de aprendizaje, siendo más relevante en el marco explicativo de esos resultados el capital socioeconómico de la familia que el factor dependencia del establecimiento (público o privado), lo que se debe a que Chile es uno de los países más desiguales de América latina (PROGRAMA DE LAS NACIONES UNIDAS PARA EL DESARROLLO -PNUD, 2017). Diversos estudios muestran desde los complejos impactos del proceso de municipalización en la segregación y desigualdad social (VALENZUELA; BELLEI; DE LOS RÍOS, 2014; BELLEI, 2013; BELLEI; CABALIN; ORELLANA, 2014; OCDE, 2017a), hasta la dificultad de insistir en este camino

Aproximadamente cada SLE reunirá 4 o 5 comunas o más, de forma que los 346 DAEM actuales se concentrarán en 70 SLE. Presidencial para la Calidad de la Educación fue la instancia definida por el Gobierno como salida a la crisis generada por e movimiento estudianti del año 2006. Su informe final es insustituible para comprender e contexto y dimensiones del problema tratado. dada la organización del Gobierno Local y el papel preponderante del Alcalde, que debilita el desarrollo institucional, lo que se ha llamado la “alcaldización” de la educación pública (ELACQUA; MARTÍNEZ; ANINAT, 2010; DONOSO, 2016).

La crisis de la educación pública chilena fue definida por el Consejo Asesor ${ }^{3}$ (2006), señalando que a partir de los impactos de la municipalización en los cuales la institucionalidad de la educación pública ha sido particularmente impropia, como la eficiencia, equidad y calidad de los resultados educativos, sobre los cuales, si bien no existe pleno consenso, hay claridad en términos de que "el esquema institucional ha mostrado limitaciones, vacíos e inconsistencias” (CHILE, 2006, p. 111). 
El Consejo concuerda que el desempeño del actual sistema de administración municipal de la educación es negativo. Hay acuerdo en un conjunto de condiciones que explican las falencias del sistema municipal, un financiamiento insuficiente para las obligaciones que poseen, disolución de responsabilidades entre el Ministerio y los municipios, escasa transferencia de competencias a las escuelas, relación desigual entre el sector municipal y el particular subvencionado. Algunos consejeros señalan que también influye el tamaño y las capacidades de gestión entre ellos. (CHILE, 2006, p. 115)

Previamente, un informe de la OCDE (2004) sobre la situación educativa chilena expresó la inconveniencia del modelo de municipalización por su asociación al financiamiento por subsidio a la demanda, y la preeminencia de este criterio, a la hora de las decisiones finales por sobre las razones educacionales. Ampliando la discusión del problema al sistema de financiamiento de la educación.

Las debilidades administrativas de las regiones y municipalidades
y la ambigüedad funcional en la división de responsabilidades
a través de los niveles administrativos perjudican una entrega
más efectiva de educación de calidad a los niños socialmente
marginados. (OCDE, 2004, p. 279)

También el informe expresó la traba que significan las escasas atribuciones de las autoridades subnacionales (regionales y provinciales) del Ministerio de Educación ante sus autoridades nacionales (OCDE, 2004, p. 280).

El Ministerio de Educación -desde1980- desconcentró gradualmente sus funciones en las unidades territoriales, pero con un desbalance de poder/responsabilidad entre las atribuciones del servicio central y las delegadas a las unidades subcentrales. Lo que se expresa en que el Ministerio define planes nacionales y subnacionales sin la expresa necesidad de interactuar con los municipios, que son los responsables y operadores de los establecimientos escolares públicos, y también impulsando negociaciones con los gobiernos regionales, aunque no existe la práctica regular de construir un diseño de planificación de carácter participativo con los entes subnacionales, sino a partir de las propuestas del Ministerio central (MARCEL; RACZYNSKI, 2009; DAVIS; DONOSO, 2016).

La búsqueda de solución a los principales problemas del sistema educativo sin salir de la lógica de mercado dio lugar a contradicciones relevantes en el sector, las que se generaron al buscar compatibilizar iniciativas correctivas a los instrumentos de mercado con políticas de financiamiento de mercado (COX, 2003), experimento que no fructificó 
en términos generales, por cuanto las medidas pro mercado han sido dominantes en educación, confirmando con ello la prevalencia del paradigma económico neoliberal en este ámbito (ATRIA, 2014; BELLEI, 2015).

En este cuadro, algunos grupos conservadores insisten en mantener el sistema vigente (solo con DAEM), mejorando el valor del subsidio y reduciendo algunas trabas burocráticas (EYZAGUIRRE, 2012), e incluso en la visión de la nueva institucionalidad han remarcado que su cambio no es fundamental para mejorar la educación pública.

En referencia a estos elementos se han llevado a cabo los debates de la educación tras el movimiento estudiantil del año 2011, incorporando dimensiones no consideradas, como a su vez originando tensiones entre la gestión del Estado y la gestión de la educación con las demandas locales, con tópicos muy diferentes de los esquemas convencionales de agrupación de los actores políticos, que no responden del todo a los cánones más conocidos, generando nuevas situaciones relevantes en el escenario a atender (RUÍZ, 2016; GARRETÓN, 2016), e instalando la temática de la gobernabilidad del sector en entredicho (DE LA MAZA, 2016). Materias que no se limitan a situaciones puntuales o conflictos parciales, sino a elementos sustantivos sobre lo que ha hecho el Estado y lo que podría hacer bajo distintas concepciones que dicen relación con la gobernabilidad del sector (MAZZUCATO, 2014).

Para abordar los desafíos del Estado subnacional en educación es imprescindible dotarle de mejores herramientas de gestión, con una visión estratégica que supere los problemas del cortoplacismo que saturan el escenario político. El PADEM plantea una visión general de la gestión de la educación municipal; no obstante, lo importante es que en un alto porcentaje de municipios esta perspectiva no se traduce en metas estratégicas ni en plazos adecuados para lograrlas. Por otra parte, tampoco queda claramente evidenciado cómo y por qué las inversiones y actividades declaradas en el PADEM conducirán a la educación municipal de la comuna a los resultados deseados (DONOSO; BENAVIDES, 2017). Por ello no es de extrañar que algunos de los problemas y dificultades que arrastra el PADEM como instrumento de planificación sean relevantes, como lo refieren Raczynski y Salinas (2009, p. 148):

Aunque los PADEM casi siempre plantean una visión general que enmarcaría la administración/gestión de la educación municipal, en un alto porcentaje de municipios esta visión no se traduce en metas estratégicas ni en plazos estimados para lograrlas y no es evidente cómo y por qué las inversiones y actividades que enuncia el PADEM contribuirán a acercar la educación municipal de la comuna al horizonte deseado. 
Complementariamente, los autores mencionan: "El PADEM plantea metas anuales de gestión en distintas áreas [...]; por lo general, las metas tienen pobres indicadores y mecanismos de seguimiento y evaluación de sus logros" (RACZYNSKI; SALINAS, 2009, p. 182). Tales debilidades son consistentes con la institucionalidad municipal, ya que pese a los esfuerzos llevados a cabo para fortalecer sus capacidades de gestión del área educacional, éstos no han sido suficientes. En general la administración municipal presenta una débil imagen pública y se enfrenta al desafío de consolidarse como órgano impulsor del gobierno local en esta área (ZAMORANO, 2006).

A partir de lo señalado, es necesario generar un instrumento plurianual de planificación estratégica, asociado al del periodo de gobierno de los directivos locales (4 años), sustentado en un diagnóstico fundado y sólido, con un sistema de indicadores relevantes que permita establecer metas anuales y del período, hacer el seguimiento adecuado de su evolución, precisar brechas y definir requerimientos de ajustes y corrección, incluyendo el ámbito presupuestario (DONOSO; BENAVIDES, 2017).

En razón de lo expuesto, el objetivo del trabajo es disponer de indicadores que aporten información sustantiva a los instrumentos de planificación estratégica que tanto los DAEM como también los nuevos Servicios Locales de Educación Pública utilicen en este campo con la finalidad de aumentar significativamente la eficiencia de su gestión. Siguiendo a Armijo (2011, p. 8-11),

[...] cuando hablamos de planificación estratégica nos estamos
refiriendo a las grandes decisiones, al establecimiento de los
Objetivos Estratégicos que permiten materializar la Misión y la
Visión [...] La planificación estratégica en el ámbito público es una
herramienta que nos ayudará al establecimiento de prioridades,
objetivos y estrategias como apoyo a la definición de los recursos
que necesitamos para lograr los resultados esperados, por lo
tanto debe ser un proceso simple e incorporado en la rutina de
la toma de decisiones directivas en el proceso presupuestario.
Desde esta perspectiva, debemos contar con estándares de
confiabilidad para identificar aspectos claves que apoyen la gestión
organizacional.

En esta línea, y de acuerdo a Rumelt (2011), lo central del trabajo del estratega es descubrir los factores cruciales de una situación y diseñar un camino para coordinar las acciones destinadas a enfrentarles. Consistentemente, los indicadores son elementos que van a permitir medir el impacto y obtener una apreciación confiable del desempeño de la estrategia. Su aplicación debiese mejorar la gestión, y por ende impactar en el proceso para que sea más eficiente y de calidad, 
precisamente lo que se necesita y demanda a las unidades de gestión local de la educación pública chilena desde hace un tiempo.

En el sector educación existen diversos modelos de trabajo que se asemejan en sus áreas de acción, de los que pueden derivarse indicadores sustantivos. El modelo de gestión escolar de la Fundación Chile (2015) define cinco áreas: (i) Convivencia y orientación hacia los estudiantes, familias y comunidad educativa (ii) Gestión de Competencias Profesionales; (iii) Liderazgo, (iv) Gestión Curricular y Pedagógica, (v) Gestión de Resultados. Por su parte la Agencia de la Calidad de la Educación Escolar (2015), actualiza y desarrolla el modelo SACGE centrado sobre (i) Liderazgo, (ii) Gestión Pedagógica, (iii) Convivencia, (iv) Gestión de Recursos. Estas dimensiones son las que se utilizaron en el instrumento dada su pertenencia con la gestión educacional y han sido instaladas en el Marco para la buena Dirección y el Liderazgo Escolar (CHILE, 2015) y también más recientemente, en el Marco para la Buena Dirección y el liderazgo local (URIBE et al., 2017), los que se usan como referentes generales de un área que requiere de nuevos desarrollos.

El trabajo considera responder a la problemática de disponer de indicadores relevantes para la gestión de la educación pública de las unidades subnacionales de educación, sean los actuales DAEM o los SLE -hoy en implementación-, a partir de los antecedentes expuestos que en lo fundamental destacan la ausencia de este tipo de señales, su escasa relevancia, las dificultades para su trazabilidad, los problemas de recursos financieros para instalar sistemas de control más complejos, y por ende, los impactos en la gestión local que implican no disponer de este tipo de herramientas en forma oportuna, adecuada y pertinente con elementos educacionales claves para el desarrollo estratégico de los sistemas locales de educación.

El sentido de esta propuesta es contribuir a incorporar la planificación estratégica de la educación como política de Estado de mediano y largo plazo y como factor esencial de desarrollo impactando a los territorios más necesitados.

\section{LA ORGANIZACIÓN DE LA EDUCACIÓN PUBLICA CHILENA}

La reforma de 1980 (CHILE, 1980) y posteriormente la Ley Orgánica Constitucional de Enseñanza (LOCE) (CHILE, 1990a) y la de organización del Ministerio de Educación (CHILE, 1990b), fueron las principales leyes sectoriales del régimen autoritario y dieron cuerpo a la institucionalidad actual, sumándose -en democracia-la Ley General de Educación (CHILE, 2009)- que sustituyó a la LOCE y luego, la del Sistema Nacional de Aseguramiento de la Calidad (CHILE, 2011), y la reciente ley creada por el Sistema de Educación Pública (CHILE, 2017a). 
Adicionalmente es indispensable comprender que Chile es uno de los países de mayor centralización del Estado ${ }^{4}$, pese a los esfuerzos que se han venido haciendo en materia de descentralización y desconcentración (OCDE, 2017b). Si bien Chile a mediados de la década de los 70' empezó un proceso de descentralización, que ha avanzado lentamente, incluso tras el retorno de la democracia, el Ministerio de Educación en el nivel central ha conservado gran parte de sus atribuciones, desconcentrando hacia los territorios regionales algunas funciones menores.

Las principales transformaciones basales se generaron en la reforma de 1980. El sistema escolar de provisión mixta fortaleció decididamente a los privados -como fue consignado previamente-, y en la actualidad el sector particular subvencionado (55\%) es el principal operador (2018), seguido por el sector público (36\%), y a distancia por el particular pagado.

La organización del sector público, tras la ley de Aseguramiento de la Calidad (2011), entregó al Consejo Nacional de Educación (CNED) las atribuciones superiores de regulación y gobernanza del sistema. El Ministerio de Educación le reporta al CNED las materias curriculares (el país tiene planes y programas curriculares de carácter obligatorio) y de organización general del régimen escolar (ciclo de educación parvularia, enseñanza básica y media).

Algunas funciones que otrora cumplía el Ministerio de Educación, la ley en comento las distribuyó en dos nuevas entidades públicas independientes. Aquellas materias referidas a la evaluación de los aprendizajes de los estudiantes son propios de la Agencia de Calidad (responsable de las pruebas nacionales e internacionales), como a su vez se creó la Superintendencia de Educación Escolar para el control de las normas financieras, laborales y operacionales de los establecimientos escolares (públicos y privados) y de la gestión de los DAEM y SLE.

En materia de financiamiento escolar, desde el año 1981 a la fecha se aplica el sistema de subsidio a la demanda que se entrega al sostenedor (privado o público), a partir de la asistencia promedio mes de los estudiantes al establecimiento, según criterios de valuación (por nivel: parvulario, básico, medio; y un factor adicional de complejidad: necesidades especiales de aprendizaje, de formación técnica o preferencial por pobreza). Lo cierto es que todo proceso de financiación sigue el sistema de subsidio, excepto el financiamiento del equipo técnico de los nuevos SLE y sus gastos operacionales.

La reforma de 1980 traspasó a los Municipios todos los establecimiento públicos, los que hasta entonces eran responsabilidad del Ministerio de Educación. De forma que a partir de esa fecha el único responsable operacional de la educación pública es el municipio (en cada una de las 346 comunas). Éste no tiene dependencia directa del Ministerio,

4 Delamaza (2016) señalan que históricamente el país ha tenido una fuerte visión centralista, complementada con el hecho que los actores regionales han alcanzado un escaso impacto en la matriz sociopolítica del país. 
salvo en materia curricular y algunas normativas básicas, por ende el Jefe DAEM -en los hechos- puede relacionarse con la institucionalidad pública central del Ministerio sin pasar por la feble institucionalidad subnacional. Esta situación se ha mantenido e incrementado con las nuevas funciones de los SLE. De allí la importancia de optimizar los procesos de gestión y esencialmente los relacionados con la formación de los estudiantes y sus aprendizajes (gestión del currículo).

Si bien en cada Municipio el responsable final de la educación es el Alcalde (sostenedor), corresponde al Jefe DAEM ${ }^{5}$-cargo inicialmente de confianza del Alcalde-, dirigir el día a día del sector. El Jefe DAEM debe guiar la elaboración del Plan Anual de Desarrollo Educativo (PADEM) y también responsabilizarse de sus resultados. Si bien el Plan requiere de la aprobación de los integrantes del Consejo Municipal, siguiéndose ciertos procedimientos y plazos, su normativa no regula precisamente los indicadores que debe adoptar, más allá de aquellos que normativamente define la Agencia de Calidad (CHILE, 2014c), esencialmente referidos a los aprendizajes de los estudiantes en cada nivel específico del sistema (DONOSO, 2018).

Los DAEM conforman realidades altamente dispares. Un 65\% posee 8 o menos establecientes escolares, con una matrícula total inferior a los 2 mil estudiantes, gran parte de ellos de enseñanza básica y con cuerpos docentes de 70 o menos educadores. En tanto, en el otro extremo, un reducido número de comunas (15\%) posee sistemas públicos con más de 15 mil estudiantes y 800 docentes aproximadamente (CHILE, 2017b). No obstante estas diferencias, sus responsabilidades en el proceso educativo son las mismas. Los tamaños de las unidades impactan directamente los recursos económicos y humanos disponibles, de allí la importancia que los indicadores que se empleen sean relevantes (dirigidos a dimensiones claves); adecuados, esto es que la información pueda obtenerse y procesarse de manera simple y a bajo costo, y finalmente sean pertinentes, es decir, entreguen información que oportunamente ilumine la situación del momento y sus tendencias, para que puedan adoptarse las correcciones requeridas.

Un segundo problema en esta línea es que el principal instrumento de planificación de los establecimientos educacionales, el Proyecto de Mejoramiento Educativo, que es la carta de navegación de cada centro escolar, no dialoga con el PADEM. Es decir, no existe una articulación específica entre ambos instrumentos, y por tanto coexisten

Si bien actualmente el cargo se selecciona por el Sistema de Alta Dirección Pública, el Alcalde posee plenas atribuciones para designar a cualquier integrante de la terna quina final o bien declarar desierto el concurso pese a que no se complementan. En razón de ello, es fundamental definir indicadores que sean vinculantes (compartidos) por ambos instrumentos, para articularles y potenciar su aporte.

El trabajo pone a disposición de las unidades de gestión local, DAEM y SLE, indicadores relevantes para la gestión de la educación pública de las unidades subnacionales, que permitan a sus equipos 
directivos tener un seguimiento efectivo de sus objetivos y metas, y determinar las brechas de cumplimiento, a fin de adoptar decisiones oportunas que optimicen su labor, materia que en la educación pública chilena es de carácter estratégico, dada la situación en que se encuentra tras casi cuatro décadas de compleja existencia.

\section{METODOLOGÍA}

Los datos que se exponen corresponden a un estudio exploratorio/ descriptivo de carácter cualitativo (desarrollado el año 2017), que se enfoca a precisar y relevar indicadores sustantivos de las unidades de gestión local de la educación pública, con la finalidad de proponer un marco de indicadores agrupados en dimensiones relevantes para estas unidades, con la finalidad de mejorar los procesos de gestión y en razón de ello, el cumplimiento de sus objetivos.

Primeramente se realizó una revisión documental de los principales indicadores de gestión utilizados en los PADEM del año $2015^{6}$ de las catorce (14) comunas del país con mayor cantidad de estudiantes en el sistema público (Arica, Iquique, Antofagasta, Copiapó, La Serena, Coquimbo, Santiago, Pudahuel, Puente Alto, San Bernardo, Peñalolén, Concepción, Valdivia, Osorno). Estos planes son públicos, por ende se accede a ellos sin restricciones. Se identificaron y analizaron sus indicadores recurrentes, se clasificaron y agruparon en función de su finalidad.

Los criterios de selección fueron de frecuencia, es decir, aquellos más utilizados en los PADEM, conforme los marcos referenciales (Marco para la Buena Dirección y el Liderazgo Escolar en el Marco de la Gestión Local, ambos del Ministerio de Educación y el Modelo de la Fundación Chile). Se buscó simplificar e integrar las dimensiones en cuatro aspectos:

(i) financiera y gestión del desempeño; (ii) Beneficiarios - Estudiantes; (iii) Gestión Pedagógica; y (iv) Resultados, formación y desarrollo.

En segunda instancia, los indicadores fueron validados $\mathrm{y}$ jerarquizados por ocho (8) responsables superiores de los Departamentos de Administración de la Educación Municipal elegidos al azar entre los 14 municipios (se conserva su anonimato para efectos del estudio), mediante una pauta de aplicación individual donde se presentan ordenados al azar, para que sean jerarquizados (se le asigna un puntaje de 1 a i=n, según la relevancia que el entrevistado le asigna), tras lo cual se procesan los resultados y se establece el valor promedio simple, el cual se ordena según recurrencia de importancia, derivándose la propuesta de indicadores debidamente jerarquizados. El diseño seguido implicó (véase Cuadro $\mathrm{N}^{\circ} 1$ ): 
CUADRO 1

RELACIÓN OBJETIVOS - PROCEDIMIENTO METODOLÓGICO

\begin{tabular}{|l|l|}
\hline \multicolumn{1}{|c|}{ OBJETIVO } & \multicolumn{1}{|c|}{ PROCEDIMIENTO } \\
\hline $\begin{array}{l}\text { (i) Identificar, producto de la revisión } \\
\text { bibliográfica, los indicadores más frecuentes } \\
\text { utilizados en los DAEM en los ámbitos } \\
\text { definidos. }\end{array}$ & $\begin{array}{l}\text { Para lo cual se seleccionaron los } \\
\text { PADEM del año 2015 de las dieciocho } \\
\text { comunas con sistemas de educación } \\
\text { pública más grandes en materia de } \\
\text { estudiantes. }\end{array}$ \\
\hline $\begin{array}{l}\text { (ii) Seleccionar los indicadores claves para } \\
\text { crear un modelo para gestionar la educación }\end{array}$ & $\begin{array}{l}\text { Analizar los indicadores, clasificarlos, } \\
\text { ponderarlos según mención y en } \\
\text { función de ello determinar los } \\
\text { imprescindibles (o suficientes) y los } \\
\text { complementarios }\end{array}$ \\
\hline $\begin{array}{l}\text { (iii) Validarles y jerarquizarlos en función de } \\
\text { su importancia, a partir de la experiencia de } \\
\text { los actores claves consultados. }\end{array}$ & $\begin{array}{l}\text { Mediante una pauta aplicada a } 8 \\
\text { Directores DAEM de las comunas } \\
\text { anteriores seleccionados al azar. }\end{array}$ \\
\hline $\begin{array}{l}\text { (iv) Proponer los indicadores estratégicos } \\
\text { según la validación. }\end{array}$ & \begin{tabular}{l} 
Propuesta final \\
\hline
\end{tabular}
\end{tabular}

Fuente: Elaboración propia.

Previamente, la pauta fue validada con un protocolo ad hoc, con un grupo equivalente de participantes (ex jefes DAEM), que no participaron en el estudio final, incorporándose sus sugerencias al instrumento definitivo. Este proceso consistió en determinar si eran relevantes, si se comprendían, si les asignaban el carácter de imprescindibles, a saber, fundamentales y claves para todo sistema local, o de complementarios, definidos como aquellos que era útil tenerlos en consideración pero que no eran vitales directamente para el sistema.

La pauta definitiva sometida a evaluación de los ocho Jefes DAEM, DAEM, fue (Cfr. Cuadro 2): 


\begin{tabular}{|c|c|c|c|}
\hline $\begin{array}{l}\text { INDICADORES } \\
\text { INDISPENSABLES }\end{array}$ & $\begin{array}{l}N^{\circ} \text { IMPORTANCIA QUE } \\
\text { ASIGNA AL INDICADOR }\end{array}$ & $\begin{array}{c}\text { INDICADORES } \\
\text { COMPLEMENTARIOS }\end{array}$ & $\begin{array}{l}\text { No IMPORTANCIA QUE } \\
\text { ASIGNA AL INDICADOR }\end{array}$ \\
\hline $\begin{array}{l}\text { Resultados Prueba } \\
\text { de Selección a la } \\
\text { Universidad (PSU) }\end{array}$ & & $\begin{array}{l}\text { Tasa de participación de } \\
\text { desarrollo de habilidades }\end{array}$ & \\
\hline $\begin{array}{l}\text { Resultados Pruebas del } \\
\text { Sistema de Medición } \\
\text { de la Calidad de la } \\
\text { Educación(SIMCE) }\end{array}$ & & $\mathrm{N}^{\circ}$ de visitas al aula & \\
\hline \% de aprobación & & $\begin{array}{l}\text { \% de establecimientos } \\
\text { clasificados según agencia } \\
\text { de calidad. }\end{array}$ & \\
\hline Tasa de retiro & & $\begin{array}{l}\text { Tasa de colegios en } \\
\text { convenio de desempeño. }\end{array}$ & \\
\hline $\begin{array}{l}\text { Índice de Vulnerabilidad } \\
\text { del establecimiento }\end{array}$ & & $\begin{array}{l}\text { Porcentaje de participación } \\
\text { en programas de salud. }\end{array}$ & \\
\hline $\begin{array}{l}\text { Tasa de cobertura } \\
\text { curricular }\end{array}$ & & $\begin{array}{l}\text { Índice de vulnerabilidad de } \\
\text { los padres }\end{array}$ & \\
\hline Tasa de matrícula & & $\begin{array}{l}\% \text { de establecimientos } \\
\text { clasificados según } \\
\text { desarrollo personal y social }\end{array}$ & \\
\hline Tasa de repitencia & & $\begin{array}{l}\text { Nivel de satisfacción de los } \\
\text { padres y/o apoderados }\end{array}$ & \\
\hline Tasa de asistencia & & $\begin{array}{l}\text { Porcentaje de utilización } \\
\text { de Centro de recursos de } \\
\text { aprendizaje }\end{array}$ & \\
\hline $\begin{array}{l}\% \text { de profesionales de } \\
\text { apoyo educativo }\end{array}$ & & $\begin{array}{l}\text { Número de registros de } \\
\text { violencia escolar }\end{array}$ & \\
\hline $\begin{array}{l}\% \text { de participación de } \\
\text { padres y apoderados }\end{array}$ & & $\begin{array}{l}\% \text { de implementación de } \\
\text { prácticas pedagógicas }\end{array}$ & \\
\hline $\begin{array}{l}\% \text { de estudiantes } \\
\text { becados }\end{array}$ & & $\begin{array}{l}\text { Y de establecimientos } \\
\text { autónomos }\end{array}$ & \\
\hline $\begin{array}{l}N^{\circ} \text { de horas contratadas } \\
\text { para docencia }\end{array}$ & & Otro cual... & \\
\hline Tasa de retención & & & \\
\hline $\begin{array}{l}\text { Tasa de éxito oportuno y } \\
\text { éxito total }\end{array}$ & & & \\
\hline Otro cual... & & & \\
\hline
\end{tabular}

Fuente: Elaboración propia a partir de la revisión documental.

\section{PRESENTACIÓN DE RESULTADOS}

La exposición de los resultados en esta primera fase se estructuró en función de las dimensiones derivadas de los marcos referenciales definidos, los que se centran en: liderazgo, convivencia escolar, gestión pedagógica y gestión de recursos. A partir de ello se identifican los indicadores más utilizados por los DAEM en sus PADEM, seleccionándose los ocho indicadores de mayor recurrencia en razón del principio de parsimonia que se busca aplicar. ${ }^{7}$

La primera área, Liderazgo (que considera la dirección unipersonal y del equipo directivo) con 122 datos, destaca como el indicador más

7 dado lo extenso de ese trabajo, no se detallan los resultados parciales, solamente se sintetizan los finales. 
frecuente: Resultados PSU (Prueba de Selección a la Universidad), seguido por Resultados SIMCE (Pruebas estandarizadas de aprendizaje escolar aplicadas censalmente), Tasa de aprobación de estudiantes (anual), IVE del establecimiento ${ }^{8}$ y Tasa de retiro de estudiantes (anual). Los municipios les asocian a factores de éxito y por ende es el reflejo de cómo se está llevando a cabo el liderazgo en el establecimiento.

Los resultados en las pruebas SIMCE y en la Prueba Nacional de Ingreso a las Universidades (PSU) son parámetros empleados regularmente para establecer ránquines de calidad (su uso en ese plano) y se han instalado como indicadores de calidad de los establecimientos, más allá de su real aporte, por lo cual son “obligatorios” en términos que no se pueden omitir como datos de importancia. Los restantes indicadores son parte de la familia de la eficiencia interna del sistema escolar y se refieren a los estudiantes que son promovidos de nivel o curso y que aprueban. Finalmente, el IVE es un factor que mide vulnerabilidad de la población escolar y es clave para determinar los pisos (y eventualmente techos) posibles de alcanzar en algunas variables, como asimismo los recursos adicionales que se asignan a los establecimientos.

En lo que se refiere al área de Gestión Pedagógica (que considera la organización de las actividades lectivas), con 63 respuestas, acusa los resultados más frecuentes: Porcentaje de estudiantes becados, Tasa de Participación en Talleres de Desarrollo de Habilidades (escolares), Número de Visitas al Aula (del equipo directivo) y Tasa de Cobertura Curricular del Establecimiento. Estos indicadores muestran que existen factores diferentes de los académicos que inciden desde el punto de vista pedagógico en el desarrollo del estudiante, que deben ser gestionados en esta dimensión. Las visitas al aula por el equipo directivo, hoy instaladas en el sistema de aseguramiento de la calidad como una obligación derivada de la ley del nuevo estatuto docente, son definidas como un factor clave en el hacer del establecimiento escolar, como de igual forma lo es la cobertura curricular. Esto es, cuánto (contenido, unidades, materias) del programa oficial se encuentra desarrollado (cubierto) en el hacer de la escuela en las diversas asignaturas y es un factor correlacionado positivamente con los resultados de los establecimientos escolares en materia de logros en las pruebas nacionales (SIMCE).

En cuanto al área de convivencia escolar (las normas, pautas y Educacional, se aplica a cada establecimiento (anualmente) para clasificarle en función de un factor compuesto de vulnerabilidad que además se vincula con beneficios que se otorgan a los estudiantes, materiales de estudio, alimentación y otros (CHILE, 2018) prácticas de comportamiento de los actores y comunidad educativa), con 53 respuestas, los indicadores dominantes fueron: Porcentaje de Participación de Padres y/o Apoderados en Actividades de Formación, y Porcentaje de Implementación del Manual de Convivencia Escolar. Asimismo, destacan luego: Nivel de Escolaridad de los Padres, y Porcentaje de Participación en Redes de Apoyo Comunales. Estos indicadores dan cuenta de la importancia del área en los establecimientos escolares, pues actualmente es obligación tener avances en esta 
materia de manera de fortalecer el proceso educativo, involucrando a los diferentes estamentos de la comunidad educativa, especialmente a padres y apoderados. La incorporación en forma activa de estos últimos al establecimiento escolar ha sido señalada como un factor importante (WEINSTEIN; MUÑOZ, 2012), que se asocia -de preferencia- al mayor nivel de escolaridad de los padres (MONTECINOS et al., 2015).

En referencia a la gestión de recursos (manejo de infraestructura, equipamiento, personal y componentes financieros), con 101 respuestas evidencia como indicadores más frecuentes: Tasa de Matrícula (cobertura territorial), Tasa de Asistencia de los estudiantes (diaria), y Porcentaje de Profesionales de la Educación de Apoyo a la Docencia que son perfeccionados. Destaca también el indicador Número de Horas Contratadas para Docencia. Dado que el sistema financiero chileno se sustenta en la subvención basada en la obtención de recursos por la asistencia media (mensual) de sus estudiantes al establecimiento, ello incide más aún en el funcionamiento del aporte financiero de gratuidad, por lo tanto cobra sentido que los indicadores que se destacan sean tasa de matrícula y tasa de asistencia, pues son los que más impactan en la generación de recursos financieros de los establecimientos escolares y en función de ello dominan por sobre otros indicadores. ${ }^{9}$

A partir de los resultados reseñados se formuló la Propuesta de Indicadores y su validación por expertos. La pauta en cuestión se aplicó a los ocho expertos considerados (identificados con la letra C), quienes jerarquizaron sus respuestas en referencia a Indicadores Imprescindibles (necesario u obligatorio), y luego a complementarios (cualidad o circunstancia que se añade a otra para hacerla íntegra). En el cuadro siguiente se muestran los resultados:

\section{CUADRO 3}

ASIGNACIÓN DE IMPORTANCIA A LOS INDICADORES IMPRESCINDIBLES

\begin{tabular}{|c|c|c|c|c|c|c|c|c|c|c|}
\hline \multirow{2}{*}{ INDICADORES INDISPENSABLES } & \multicolumn{8}{|c|}{ RESPUESTAS DE EXPERTOS } & \multirow{2}{*}{$\begin{array}{c}\text { MEDIA } \\
\text { ARIT. }\end{array}$} & \multirow{2}{*}{$\begin{array}{l}\text { POSICIÓN } \\
\text { FINAL }\end{array}$} \\
\hline & $\mathrm{C} 1$ & $\mathrm{C} 2$ & C3 & $\mathrm{C} 4$ & C5 & $\mathrm{C} 6$ & C7 & C8 & & \\
\hline 1. Tasa de matrícula (cobertura Territorial) & 2 & 8 & 5 & 3 & 3 & 1 & 6 & 7 & 4.34 & $1^{\circ}$ \\
\hline 2. Porcentaje de aprobación de estudiantes anuales & 7 & 2 & 11 & 2 & 5 & 4 & 4 & 2 & 4.65 & $2^{\circ}$ \\
\hline 3. Resultados SIMCE & 10 & 1 & 1 & 9 & 7 & 5 & 1 & 5 & 4.87 & 3은 \\
\hline 4. Tasa de cobertura curricular & 5 & 7 & 3 & 1 & 13 & 9 & 2 & 1 & 5.13 & $4 \div$ \\
\hline 5. Tasa de asistencia & 3 & 11 & 4 & 4 & 4 & 2 & 10 & 8 & 5.75 & 5은 \\
\hline 6. Tasa de repitencia & 12 & 9 & 15 & 5 & 9 & 3 & 7 & 3 & 7.88 & 60 \\
\hline 7. Resultados PSU (Prueba de Selección Universitaria) & 11 & 17 & 2 & 8 & 6 & 6 & 9 & 6 & 8.13 & $7^{\circ}$ \\
\hline 8. Tasa de retiro de estudiantes anuales & 8 & 10 & 10 & 16 & 10 & 7 & 5 & 4 & 8.75 & 80 \\
\hline 9. Tasa de retención de estudiantes & 9 & 13 & 6 & 10 & 12 & 8 & 3 & 12 & 8.88 & 9웅 \\
\hline 10. Índice de Vulnerabilidad del establecimiento & 6 & 16 & 7 & 6 & 1 & 15 & 8 & 13 & 9.00 & $10^{\circ}$ \\
\hline 11. Porcentaje de profesionales de apoyo educativo & 4 & 3 & 14 & 11 & 14 & 12 & 15 & 11 & 10.50 & $11^{\circ}$ \\
\hline 12. $N^{\circ}$ de horas contratadas para docencia & 1 & 4 & 12 & 14 & 15 & 11 & 11 & 9 & 10.87 & $12^{\circ}$ \\
\hline 13. Porcentaje de participación de padres y apoderados & 14 & 12 & 13 & 12 & 2 & 13 & 14 & 10 & 11.25 & $13^{\circ}$ \\
\hline 14. Tasa de éxito oportuno y éxito total & 15 & 15 & 16 & 15 & 8 & 10 & 13 & 17 & 13.63 & $14 \div$ \\
\hline 15. Porcentaje de estudiantes becados & 13 & 14 & 17 & 13 & 11 & 14 & 12 & 14 & 14.75 & $15 \circ$ \\
\hline
\end{tabular}

Fuente: Elaboración propia a partir de respuestas de entrevistados.

Los recursos financieros para solventar el

equipo técnico de los DAEM provienen de los recursos generados por la asistencia de los estudiantes. La Ley que rige a los SLE es diferente en este ámbito, pues provee un financiamiento directo para el personal directivo-administrativo del SLE, de acuerdo a una planta base definida. 
En el análisis del Cuadro 3, Indicadores Imprescindibles, se destacan nítidamente aquellos referidos a la eficiencia interna del sistema escolar (tasas de matrícula, de asistencia, de promoción y de retención) y también de eficiencia externa de su hacer, esencialmente derivados de resultados en las diversas pruebas SIMCE y en las de admisión a la Universidad, ${ }^{10}$ que conforman los parámetros regulares bajo los cuales se evalúa el desempeño de los establecimientos educacionales y por tanto de la gestión local, incluso con prescindencia de los recursos disponibles para acometer esta tarea por los municipios, aunque en el caso SIMCE se construyen grupos comparativos afines.

No obstante, si bien en forma regular se señala que no es posible concentrarse solamente en tales indicadores y se tiene conciencia que estos índices son en parte reduccionistas, es decir, se limitan a una dimensión importante pero insuficiente para formarse una visión completa, pese a ello tampoco se puede prescindir de ellos -entre otros motivos- porque todo el conjunto de señales del sistema educacional chileno los considera su piedra angular, derivando algunos estímulos económicos a los docentes y los establecimientos escolares que se fundan en los resultados SIMCE como componente fundamental.

Pese a lo señalado, la tasa de cobertura curricular es un indicador que puede ser aplicado en forma sistemática y que permite un control relevante del hacer del establecimiento y del subsistema local; en función de su grado de avance, puede generar desde los inicios del año escolar estrategias destinadas a su seguimiento y mejoramiento, con impactos sustantivos en los logros de los aprendizajes (CHILE, 2014b). Por otra parte, el uso de indicadores de eficiencia interna del centro escolar referidos a estudiantes (asistencia, repitencia, retiro y abandono), para ser adecuadamente dimensionados, necesita de los factores de eficiencia externa para su ponderación, pues en caso contrario se pueden producir distorsiones referidas a baja o sobre exigencia, la que puede apreciarse desde los resultados de las pruebas nacionales de logros (que están controladas por factores como nivel socioeconómico, tipo de establecimiento escolar, etc.).

A partir de la aplicación de los indicadores reseñados, mediante series de tiempo (mensuales, quincenales y anuales), la mayor parte de los cuales son de más fácil captura, registro, control, seguimiento y presentación, lo que habilita su utilización con bajos requerimientos técnicos por las unidades locales para acceder a esta información que es sustantiva y, en razón de ello, sistematizarla debidamente.

En el Cuadro siguiente $\left(\mathrm{N}^{\circ} 4\right)$ se ponderan los indicadores referidos a los aspectos complementarios: 
ASIGNACIÓN DE IMPORTANCIA DEL INDICADOR COMPLEMENTARIO

\begin{tabular}{|c|c|c|c|c|c|c|c|c|c|c|}
\hline \multirow{2}{*}{ INDICADORES COMPLEMENTARIOS } & \multicolumn{8}{|c|}{ RESPUESTAS } & \multirow{2}{*}{$\begin{array}{l}\text { MEDIA } \\
\text { ARITM. }\end{array}$} & \multirow{2}{*}{$\begin{array}{c}\text { POSICIÓN } \\
\text { FINAL }\end{array}$} \\
\hline & $\mathrm{C} 1$ & $\mathrm{C} 2$ & C3 & C4 & C5 & C6 & $\mathrm{C7}$ & $\mathrm{C8}$ & & \\
\hline 1. \% de establecimientos clasificados según agencia de calidad & 2 & 10 & 2 & 8 & 3 & 1 & 7 & 4 & 4.63 & 10 \\
\hline $\begin{array}{l}\text { 2. \% de establecimientos clasificados según desarrollo } \\
\text { personal y social }\end{array}$ & 4 & 5 & 4 & 6 & 6 & 2 & 2 & 12 & 5.13 & $2^{\circ}$ \\
\hline 3. Tasa de participación de desarrollo de habilidades & 6 & 6 & 3 & 5 & 11 & 3 & 10 & 2 & 5.75 & 3운 \\
\hline 4. Tasa de colegios en convenio de desempeño & 3 & 1 & 8 & 7 & 8 & 8 & 8 & 6 & 6.13 & $4^{\circ}$ \\
\hline 5. Tasa de retención & 7 & 7 & 12 & 2 & 2 & - & - & 3 & $6.6^{*}$ & $5 \circ$ \\
\hline 6. \% de implementación de prácticas pedagógicas & 5 & 12 & 13 & 1 & 7 & 5 & 5 & 1 & 6.25 & $6^{\circ}$ \\
\hline 7. № de registros de violencia escolar & 9 & 2 & 7 & 11 & 10 & 6 & 4 & 8 & 7.25 & $7^{\circ} \div$ \\
\hline 8. Nivel de satisfacción de los padres y/o apoderados & 10 & 3 & 6 & 9 & 4 & 11 & 9 & 7 & 7.38 & $8^{\circ}$ \\
\hline 9. $N^{\circ}$ de visitas al aula & 8 & 11 & 1 & 16 & 12 & 7 & 1 & 5 & 7.65 & $9 \circ$ \\
\hline 10. \% de establecimientos autónomos & 1 & 13 & 11 & 15 & 5 & 4 & 6 & 9 & 8.00 & $10^{\circ}$ \\
\hline 11. Índice de vulnerabilidad de los padres & 14 & 9 & 5 & 12 & 1 & 12 & 12 & 11 & 9.50 & $11^{\circ}$ \\
\hline 12. \% de utilización de CRA & 12 & 8 & 10 & 13 & 9 & 9 & 3 & 13 & 9.63 & $12^{\circ}$ \\
\hline 13. \% de participación en programas de salud & 13 & 4 & 9 & 17 & 14 & 10 & 11 & 10 & 11.0 & $13^{\circ}$ \\
\hline 14. Tasa de éxito oportuno y éxito total & 11 & - & - & 14 & - & - & - & 14 & $\mathrm{~N} / \mathrm{a}$ & - \\
\hline
\end{tabular}

Fuente: Elaboración propia a partir de respuestas de entrevistados.

En los indicadores complementarios expuestos en el Cuadro 4, se destacan la clasificación de calidad del establecimiento escolar que hace la Agencia de Calidad, ${ }^{11}$ agregando el componente desarrollo personal y social que emana de una de las dimensiones que en la actualidad se miden en las pruebas SIMCE y que han mostrado su fortaleza para comprender la interacción de otras variables relevantes del proceso formativo (CHILE, 2014a, 2014b).

En una dimensión diferente se destaca la existencia de convenios de desempeño por establecimientos escolares, donde se establecen metas en diversos ámbitos para los años de gestión. Este punto es importante, pues introduce un criterio de mayor modernidad a la gestión del establecimiento, asociada a metas y a recursos financieros. La dimensión negativa del fenómeno expuesto es que pareciera no haber un compromiso del sistema con los establecimientos y unidades escolares que no tienen buenos resultados, más allá de establecer un situación extrema que amerite el cierre del centro escolar y el traslado de los estudiantes a otro establecimiento, lo que no parece ser una solución aplicable masivamente y que se debería revisar, atendiendo que se ha demostrado como relevante el compromiso de las unidades territoriales intermedias de gestión con los centros escolares (ANDERSON, 2003; ANDERSON et al., 2012; URIBE et al., 2017).

\section{DEBATE FINAL: PROPUESTA DE INDICADORES}

El sentido de definir indicadores claves es disponer, a partir de su aplicación, de antecedentes relevantes, cualquiera sea la herramienta de gestión final bajo la cual se organicen los datos, que permitan adoptar a los establecimientos en cuatro niveles: Alto, Medio, Medio bajo e Insuficiente, al respecto véase (CHILE, 2014a). 
decisiones fundadas en forma oportuna y/o en momentos críticos o estratégicos.

Un segundo principio es que éstos sean pertinentes y sustantivos para medir procesos que conlleven el cumplimiento de los principales objetivos estratégicos de las unidades educacionales de gestión local y disponer de datos confiables y sólidos que aseguren la eficiencia del sistema.

Un tercer principio es que su operatoria sea simple y de bajo costo de gestión, de manera que puedan implementarse por unidades que no disponen de grandes recursos financieros ni humanos para levantar, procesar y analizar esta información, es decir, que no demandan de una inversión adicional que restrinja su aplicación.

Un cuarto principio, estrechamente asociado a los anteriores, es que la información provista provea antecedentes oportunos para orientar sistemáticamente el hacer de las unidades locales de gestión de la educación y el desempeño de los agentes claves de sus establecimientos educacionales.

La propuesta que se presenta y debate define siete (7) indicadores para el área de recursos financieros, siete para la de Beneficiarios (4 correspondientes al ámbito de Liderazgo y 3 al de Convivencia Escolar), y seis para Procesos Internos, y finalmente cinco para el área de formación y desarrollo de la organización.

\section{CUADRO 5 \\ PROPUESTA DE INDICADORES DIMENSIÓN FINANCIERA/ GESTIÓN DE DESEMPEÑO}

\begin{tabular}{|l|}
\hline \multicolumn{1}{|c|}{ INDICADOR } \\
\hline 1. Tasa de matrícula (cobertura de la población en edad escolar en la comuna) \\
\hline 2. Tasa de asistencia (\% estudiantes asistan de forma regular a clases) \\
\hline $\begin{array}{l}\text { 3. Capacitación a docentes y profesionales de apoyo educativo (\% de docentes y asistentes } \\
\text { de la educación de cada establecimiento que se perfecciona por año) }\end{array}$ \\
\hline $\begin{array}{l}\text { 4. Número de horas contratadas para docencia (\% dotación docente necesaria de cada } \\
\text { escuela o comuna en relación a horas de programas de estudio) }\end{array}$ \\
\hline $\begin{array}{l}\text { 5. Porcentaje de utilización de CRA (\% de la cobertura del programa Centro de Recursos } \\
\text { de Aprendizaje). }\end{array}$ \\
\hline $\begin{array}{l}\text { 6. Número de horas contratadas para Asistentes de la educación con funciones de apoyo } \\
\text { en aula }\end{array}$ \\
\hline 7. Número de horas contratadas para profesionales de la educación no docentes \\
\hline
\end{tabular}

Fuente: Elaboración propia a partir de respuestas de entrevistados

Como se registra en la Cuadro 5, los indicadores en esta dimensión son aquellos que le permitan a la unidad local tener claridad sobre el comportamiento de las fuentes de ingresos financieros; dado el sistema de subsidio a la demanda vigente en Chile, éstos se asociarán a la matrícula de estudiantes y a su asistencia (diaria/promedio mes) al centro escolar. Los otros indicadores priorizados se relacionan con insumos (y gastos) claves del sistema, a saber: horas docentes, horas de asistentes de la educación y de otros profesionales, que junto a la 
utilización de materiales educativos (CRA), constituyen la base sobre la cual se asientan los procesos pedagógicos en el sector público.

Un factor complementario se refiere a la capacitación de los docentes que posee un claro sentido estratégico. Con estos indicadores, debidamente sistematizados y monitoreados regularmente (quincena/ mes/trimestre), la unidad de gestión puede llevar un control efectivo de su operación y disponer a tiempo de los antecedentes para corregir el rumbo, esencialmente respecto del balance ingresos/egresos, dado que los ingresos suelen ser variables, por el sistema de financiamiento señalado- en tanto los egresos son mucho más estables. Ciertamente algunos de los indicadores (matrícula) suelen tener variaciones menores, pero es vital para el sistema atender a su evolución en momentos críticos, pues los efectos de los conflictos estudiantiles (paros de estudiantes, de docentes y ocupación o "toma” de los establecimientos escolares) inciden en forma directa en la migración de matrícula pública hacia el sector privado, de manera que se necesitan procesos de evaluación continuos y más eficientes (DONOSO; BENAVIDES, 2017).

\section{CUADRO 6}

PROPUESTA DE INDICADORES DIMENSIÓN BENEFICIARIOS

\begin{tabular}{|l|l|}
\hline \multirow{5}{*}{ Liderazgo } & $\begin{array}{l}\text { 1. Porcentaje de establecimientos clasificados según } \\
\text { categoría de desempeño }\end{array}$ \\
\cline { 2 - 3 } & $\begin{array}{l}\text { 2. Porcentaje de establecimientos que implementan } \\
\text { visión estratégica compartida }\end{array}$ \\
\cline { 2 - 3 } Formación y Convivencia & $\begin{array}{l}\text { 3. Número de directores que guían, dirigen y gestionan } \\
\text { los procesos de enseñanza-aprendizaje }\end{array}$ \\
\cline { 2 - 3 } & $\begin{array}{l}\text { 4. Porcentaje de establecimientos que desarrollan } \\
\text { capacidades directivas, docentes y asistentes de la } \\
\text { educación }\end{array}$ \\
\hline & $\begin{array}{l}\text { 1. Porcentaje de implementación del Manual de } \\
\text { Convivencia Escolar en los establecimientos }\end{array}$ \\
\cline { 2 - 3 } & 2. Número de Registros de hechos de violencia escolar \\
\cline { 2 - 3 } & $\begin{array}{l}\text { 3. Porcentaje de asistencia de padres y apoderados a las } \\
\text { actividades educacionales }\end{array}$ \\
\hline
\end{tabular}

Fuente: Elaboración propia a partir de respuestas de entrevistados.

Desde la perspectiva del liderazgo (Cuadro 6) se definen los dos primeros indicadores referidos al establecimiento escolar, a saber: la clasificación según categoría de desempeño que se genera a partir de un conjunto de indicadores derivados de las pruebas nacionales, cuyas implicancias sobre la autonomía de la gestión del establecimiento son relevantes según la categoría que se alcance y que responden a los criterios de la Agencia de Calidad Escolar. La segunda agrupación (familia) de criterios es el grado de participación de los actores en el proyecto educativo, y más directamente del proceso de enseñanzaaprendizaje de acuerdo con la nueva normativa de gestión y de carrera docente, escenario en el cual también cabe el indicador final de esta sección (desarrollo de capacidades directivas). 
Estos indicadores permiten dimensionar la gestión que se realiza en cada establecimiento y proponer medidas de apoyo o correctoras que apunten a cuestiones sustantivas. La clasificación de los establecimientos que establece la Agencia de Calidad Escolar tendrá variaciones anuales, en los casos en riesgo, pero los otros indicadores están expuestos a una actualización mensual y permiten, por ende, direccionar el sistema escuela en forma oportuna.

En el ámbito de la convivencia, que complementa esta área, se precisa en primer lugar, determinar el grado de cumplimiento de las normas legales al respecto, es decir si existe un manual en este plano y si su edición ha seguido el protocolo respectivo (discusión con padres, conocimiento de la comunidad escolar), luego el seguimiento de los registros de violencia escolar y su desenlace según los protocolos definidos para ello, y finalmente, el grado de involucramiento de las familias en las actividades del centro escolar, pues la convivencia requiere la presencia de los actores en un mismo contexto/ escenario. Todos estos indicadores pueden tener una actualización quincenal/ mensual y atender un área muy sensible en la sociedad chilena, que requiere de medidas eficientes lo más próximas a la ocurrencia de los hechos iniciales.

\section{CUADRO 7}

PROPUESTA DE INDICADORES DIMENSIÓN GESTIÓN PEDAGÓGICA

\begin{tabular}{|l|l|}
\hline \multirow{4}{*}{ Gestión Pedagógica } & 1. Resultados de aprendizaje \\
\cline { 2 - 2 } & 2. Repitencia \\
\cline { 2 - 2 } & 3. Cobertura Curricular \\
\cline { 2 - 2 } & 4. Tasa Retención \\
\cline { 2 - 2 } & 5. Prácticas Pedagógicas Efectivas \\
\cline { 2 - 2 } & 6. Tasa de titulación \\
\hline
\end{tabular}

Fuente: Elaboración propia a partir de respuestas de entrevistados.

La gestión pedagógica (Cuadro 7) se estructura en función de dos criterios; primero netamente por resultados, a saber: logros de aprendizaje, tasa de repitencia anual de los estudiantes y tasa de titulación (graduación). Si bien operan estos registros en forma anual, se puede definir un seguimiento mensual de su evolución, esencialmente antes de la mitad del período escolar, y con ello prever los resultados y proyectarles, en función de los datos, según su ocurrencia en el tiempo, adoptando medidas que permitan cambiar su orientación.

La segunda dimensión se refiere a la tasa de cobertura curricular, factor que es clave para explicar los resultados en las pruebas nacionales de logros de aprendizaje y los resultados en este ámbito del establecimiento escolar, el cual se complementa con el estilo de prácticas pedagógicas implementadas, cuya incidencia sobre los aprendizajes es igualmente determinante, aunque en menor grado de la cobertura curricular. 
La incidencia de estos factores es determinante sobre los resultados de aprendizaje, de allí su importancia y trascendencia para que su actualización sea por lo menos mensual.

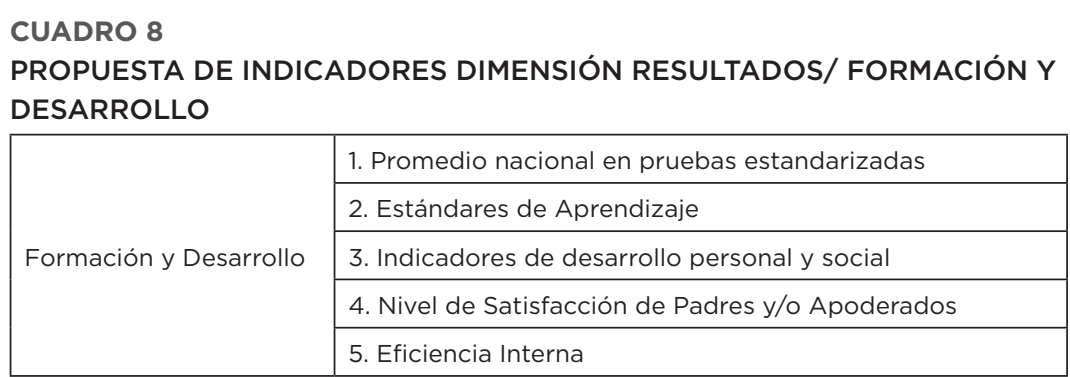

Fuente: Elaboración propia a partir de respuestas de entrevistados.

Respecto de esta última dimensión (Cuadro 8), se establece como referente la comparación de los promedios nacionales de los resultados en las pruebas nacionales, contrastada con el promedio local, según los distintos estratos de clasificación que se empleen. El sentido de esta relación es determinar con diversos factores el lugar en que se encuentra frente a sí mismo, frente a unidades iguales y ante las desiguales, para apreciar el panorama en su complejidad.

En función de lo mismo, la referencia acerca de los estándares de aprendizaje, reforzados por los indicadores de desarrollo personal (como se señaló, derivados de las pruebas nacionales), sumados a la satisfacción de los padres como factor determinante en un sistema abierto como el chileno en el que no es vinculante la localización del hogar para definir el establecimiento escolar público al que asiste el estudiante, y finalmente los indicadores de eficiencia interna del sistema (tasa de éxito oportuno y total, repitencia, promoción, retención de estudiantes y tasa de escolaridad interna).

Finalmente, teniendo claro que es indispensable identificar regularmente oportunidades de mejora en un sistema educativo, los veinticinco indicadores definidos y aplicables en períodos e intervalos sistemáticos de tiempo y del año escolar, permiten obtener una situación adecuada y suficientemente completa del acontecer del sistema local de educación local y en función de ello, de cada uno de los establecimientos de su jurisdicción. Por ende resulta fundamental atender a los procesos (cobertura curricular, aprendizajes, didáctica, etc.) y resultados educativos en forma más oportuna y adecuada de lo que se hace en la actualidad.

Su operación en el tiempo debería incrementar crecientemente la eficiencia interna y externa del sistema local de educación. Para lo cual se necesita, básicamente: (i) identificar los responsables de su medición y control; (b) determinar nacional/localmente las metas y sentido del control para cada instancia; (iii) un sistema de reportes 
de resultados accesible a las unidades y personas que necesiten dicha retroalimentación, (iv) evidencias sólidas que permitan decisiones adecuadas y oportunas.

Afortunadamente, con el apoyo de los recursos informáticos, estos requisitos pueden cumplirse de forma muy eficiente en la actualidad, si se suma a estos aspectos la familiaridad que presentan buena parte de estos indicadores para los gestores de la educación local, las experiencias que existen en el levantamiento de antecedentes, incluso diario, ${ }^{12}$ la simplicidad de su registro y procesamiento, y la relevancia de información que se provee para la toma de decisiones. Todo lo cual permite que con una baja inversión de recursos en capacitación para los responsables de estos procesos sus efectos se logren prontamente, y su impacto se puede percibir inclusive ya en el corto plazo (dos años aproximadamente) (ERRÁZURIZ; KUTSCHER; WILLIAMSON, 2016).

\section{REFERENCIAS}

ANDERSON, Stephen. The school district role in educational change: a review of the literature. International Centre for Educational Change, 2003.

ANDERSON, Stephen; MASCALL, Blair; STIEGELBAUER, Suzanne; PARK, Jaddon. No one way: differentiating school district leadership and support for school improvement. Journal of Educational Change, v. 13, n. 4, p. 403-430, 2012. DOI: 10.1007/s10833-012-9189

ARMIJO, Marianela. Planificación estratégica e indicadores de desempeño en el sector público. Santiago: Instituto Latinoamericano y del Caribe de Planificación Económica y Social (ILPES), 2011.

ATRIA, Fernando. Derechos sociales en educación: un nuevo paradigma de lo público. Santiago: LOM, 2014.

BALL, Stephen; YOUDELL, Deborah. Privatización encubierta de la educación pública. Instituto de Educación, Universidad de Londres. V ${ }^{\circ}$ Congreso Internacional de Educación, Londres, 2007.

BELLEI, Cristián. El gran experimento: mercado y privatización de la educación chilena. Santiago: LOM, 2015.

BELLEI, Cristián. El estudio de la segregación socioeconómica y académica de la educación chilena. Estudios Pedagógicos, Santiago, v. 39, n. 1, p. 325-345, 2013.

BELLEI, Cristián; CABALIN, Cristián; ORELLANA, Víctor. The 2011 Chilean student movement against neoliberal educational policies. Studies in Higher Education, v. 39, n. 3, p. 426-440, 2014.

CHILE. Ministerio de Economía, Fomento y Turismo. Ley n. 18.956, de 22 de febrero de 1990. Reestructuró el Ministerio de Educación Pública. Santiago, 1990.

CHILE. Ministerio de Educación -Mineduc. DFL n. 1-13.063, de 02 de junio de 1980. Estableció la municipalización de la enseñanza. 1980.

CHILE. Ministerio de Educación -Mineduc. Ley n. 20.370, de 12 de septiembre de 2009. Establece Ley General de Educación de Chile (LGE). Santiago, 2009.

CHILE. Ministerio de Educación -Mineduc. Ley n. 20.529, de 27 de augusto de 2011. Establece el "Sistema Nacional de Aseguramiento de la Calidad de la Educación Parvularia, Básica y Media y

12

La asistencia delos estudiantes a establecimiento escolar se realiza diariamente, en un registro de una plataforma nacional manejada por el Ministerio de Educación
CHILE. Ministerio de Educación -Mineduc. Agencia de Calidad de la Educación. Categorías de desempeño de los establecimientos educacionales. Santiago: Mineduc, 2014a.

CHILE. Ministerio de Educación -Mineduc. Estándares Indicativos de Desempeño para los

Establecimientos Educacionales y sus Sostenedores. Santiago: Mineduc, 2014b. 
CHILE. Ministerio de Educación -Mineduc. Agencia de Calidad de la Educación. Estándares indicativos de desempeño para los establecimientos educacionales y sus sostenedores. Santiago: Mineduc, 2014c.

CHILE. Ministerio de Educación -Mineduc. El marco para la buena dirección y el liderazgo escolar. Santiago: Mineduc, 2015.

CHILE. Ministerio de Educación -Mineduc. Ley n. 21.040, de 24 de noviembre de 2017. Cera el Sistema Nacional de Educación Pública. Santiago, 2017.

CHILE. Ministerio de Educación -Mineduc. Junta Nacional de Auxilio Escolar y Becas -Junaeb. Catálogo de Datos. Índice de Vulnerabilidad. Santiago de Chile, 2018. Disponible en: http://junaebabierta.junaeb.cl/catalogo-de-datos/indicadores-de-vulnerabilidad. Acceso en: 16 mar. 2018.

CHILE. Ministerio de Educación Publica. Ley n. 18.962, de 10 de marzo de 1990. Ley Orgánica Constitucional de Enseñanza (LOCE). Santiago, 1990.

CHILE. Presidencia de la República. Consejo Asesor Presidencial para la Calidad de la Educación. Informe Final. Santiago, 2006.

CHILE. Sistema Nacional de Información Municipal-SINIM. Subsecertaría de desarrollo Regional, Ministerio del Interior, 2017. Disponible on: <http:/www.subdere.gov.cl/programas/divisi\%C3\%B3nmunicipalidades/sistema-nacional-de-informaci\%C3\%B3n-municipal-sinim>. Acesso on: 22 ene. 2018.

COX, Cristián (Ed.). Políticas educacionales en el cambio de siglo: la reforma del sistema escolar en Chile. Santiago: Editorial Universitaria, 2003.

DAVIS, Giselle; DONOSO, Sebastián. Desafíos para la construcción de políticas educacionales de los gobiernos subnacionales chilenos: el caso de la Región del Maule. Jornal de Políticas Educacionais, v. 9, n. 17 e 18, p. 4-20, jan./dez. 2016.

DELAMAZA, Gonzalo. Sociedad civil, ciudadanía, movimiento social en el Chile de Hoy. In: GARRETÓN, Manuel Antonio (Comp.). La gran ruptura, institucionalidad política y actores sociales en Chile del siglo XXI. Santiago: LOM, 2016. p. 109-132.

DONOSO, Sebastián. Chilean education policy between the student movement of 2011 and reformist debates of 2014. Universum, v. 31, n. 1, p. 105-121, 2016.

DONOSO, Sebastián. La nueva institucionalidad subnacional de educación pública chilena y los desafíos de gestión para el sistema escolar. Educar em Revista, Curitiba, v. 34, n. 68, p. 29-48, mar./abr. 2018. DOI: 10.1590/01.04-4060.57480

DONOSO, Sebastián; BENAVIDES, Nibaldo. Descentralización de la gestión de la educación pública e institucionalidad local en Chile: el caso de los Directores Comunales de Educación. Innovar, v. 27, n. 64, p. 29-42, 2017.

DONOSO, Sofia. Dynamics of change in Chile: expleaining the emergency of the 2006 Pingüino. Movement: Journal of Latinamerican Studies, v. 45, n. 1, p. 1-29, feb. 2013.

ELACQUA Gregory; MARTÍNEZ, Matías; ANINAT, Cristóbal. ¿Cómo fortalecer la educación municipal? capacidad y responsabilidad política. In: MARTINIC, Sergio; ELACQUA, Gregory (Ed.) ¿Fin del ciclo? Cambio en la gobernanza del sistema educativo. Santiago: Unesco-SantiagoPUCCh, Santiago, 2010. p. 101-130.

ERRÁZURIZ, Manuela; KUTSCHER, Macarena; WILLIAMSON, Carlos. La Ley 20.501 sobre Calidad y Equidad en los Colegios Públicos: efectos de la selección de directores por la Alta Dirección Pública (ADP). CLAPESUC, (Nº 28). 2016. Disponible en: http://www.clapesuc.cl/assets/ uploads/2017/03/efectos-de-la-seleccin-de-directores-por-la-alta-direccin-pblica-adp.pdf 2016. Acceso en: 1 oct. 2017.

ESPÍNOLA, Viola; SILVA, María Ester. Competencias del sostenedor para una efectiva gestión del mejoramiento educativo en el nivel local: una propuesta. Santiago: Instituto de Políticas Públicas Expansivas, 2009.

EYZAGUIRRE, Silvia. Fortalecimiento de la educación escolar pública: ¿Desmunicipalización? Puntos de Referencia, 340. Disponible en: http://www.cepchile.cl/dms/archivo_4958_3061/ pder340_SEyzaguirre.pdf. 2012. Acceso en: marzo 21, 2014. 
FUNDACIÓN CHILE. Modelo de calidad de la gestión escolar. Santiago: Centro de Innovación en Educación de Fundación Chile, 2015.

GARRETÓN, Manuel Antonio. La ruptura entre política y sociedad: una introducción. In: GARRETÓN, Manuel Antonio (Comp.). La gran ruptura, institucionalidad política y actores sociales en Chile del siglo XXI. Santiago: LOM, 2016. p. 11-20.

GONZALEZ, Alvaro; GONZALEZ, Micaela; GALDAMES, Sergio. El sostenedor como agente de cambio: el rol de los coordinadores técnicos en el apoyo a establecimientos municipales chilenos. Pensamiento Educativo: Revista de Investigación Educacional Latinoamericana, v. 52, n. 1, p. 47-64, 2015.

JOFRÉ, Gerardo. Subvenciones en educación. Estudios Públicos, n. 32, p. 31-55, 1988.

JOIKO, Sara. El cuasi-mercado educativo en Chile: desarrollo y consecuencias. Diálogos Educativos, v. 12, n. 23, p. $148-174,2012$.

LARRAÑAGA, Osvaldo; PEIRANO, Claudia; FALCK, Denise. Una mirada al interior del sector municipal. In: MARCEL, M.; RACZYNSKI, D. (Ed.). La asignatura pendiente. Santiago Uqbar; CIEPLAN, 2009. p. 77-97.

MARCEL, Mario; RACZYNSKI, Dagmar. La asignatura pendiente: claves para la revalidación de la educación pEs icltad, dando cuenta con eloública de gestión local en Chile. Santiago: Uqbar, 2009. (CIEPLAN).

MAZZUCATO, Mariana. El Estado emprendedor. Madrid: RBE Libros, 2014.

MONTECINOS, Carmen; AHUMADA, Luis; GALDAMES, Sergio; CAMPOS, Fabián; LEIVA, María Verónica. Targets, threats and (dis)trust: the managerial troika for public school principals in Chile. Education Policy Analysis Archives, v. 23, n. 87, 2015. http://dx.doi.org/10.14507/epaa. v23.2083.

ORGANIZACIÓN PARA LA COOPERACIÓN Y EL DESARROLLO ECONÓMICO -OCDE. Chile. Revisión de las políticas Nacionales de Educación. Santiago de Chile, OCDE, 2004.

ORGANIZACIÓN PARA LA COOPERACIÓN Y EL DESARROLLO ECONÓMICO -OCDE. Educación en Chile. Evaluación de Políticas nacionales de Educación. Fundación SM para esta edición en español. Paris: OCDE, 2017a.

ORGANIZACIÓN PARA LA COOPERACIÓN Y EL DESARROLLO ECONÓMICO -OCDE. Making Decentralisation Work in Chile: Towards Stronger Municipalities, OECD Multi-level Governance Studies. Paris: OECD, 2017b.

POLITEIA. Estudio mejoramiento de la gestión y la calidad de la educación municipal. 2008. Disponible en: http://politeia.cl/proyectos/estudio-mejoramiento-de-la-gestion-y-la-calidad-de-laeducacionmunicipal. Acceso: Nov., 22 de 2009.

PROGRAMA DE LAS NACIONES UNIDAS PARA EL DESARROLLO -PNUD. Desiguales. Orígenes, cambios y desafíos de la brecha Social en Chile. Santiago: PNUD, 2017.

RACZYNSKI, Dagmar; SALINAS, Daniel. Prioridades, actores y procesos en la gestión municipal de la educación. In: MARCEL, Mario; RACZYNSKI, Dagmar. (Ed.). La asignatura pendiente: claves para la revalidación de la educación pública de Gestión Local en Chile. Santiago: Uqbar. 2009. p. $135-176$.

RUIZ, Carlos. Crisis política en Chile: neoliberalismo, cambios sociales y democracia. In: GARRETÓN, Manuel Antonio (Comp.). La gran ruptura, institucionalidad política y actores sociales en Chile del siglo XXI. Santiago: Lom, 2016. p. 83-108.

RUMELT, Richard. Good strategy/bad strategy: the difference and why it matters. Crown Publishing, CA, 2011.

URIBE, Mario; BERKOWITZ, Daniela; TORCHE, Pablo; GALDAMES, Sergio. Marco para la gestión y el liderazgo educativo local: desarrollando prácticas de liderazgo intermedio en el territorio. Valparaíso, Chile: Lideres Educativos, Centro de Liderazgo para la Mejora Escolar, 2017.

VALENZUELA, Juan Pablo; BELLEI, Cristián; DE LOS RÍOS, Danae. Socio-economic school segregation in a market oriented educational system: the case of Chile. Journal of Education Policy, v. 29, n. 2, p. 217-241, 2014. 
WAISSBLUTH, Mario. Cambio de rumbo: una nueva vía chilena a la educación. Santiago: Ramdom House Mondadori S.A., 2013.

WEINSTEIN, José; MUÑOZ, Gonzalo ¿Qué sabemos sobre los directores de escuelas en Chile? Santiago: Fundación Chile; Pontificia Universidad Católica de Chile, 2012.

ZAMORANO, Luis. Descentralización de la educación y el desempeño de los municipios. Santiago: Instituto Chileno de Estudios Municipales (ICHEM), 2006. (Documento, 21). 